\title{
Neonatal pneumatosis cystoides intestinalis caused by volvulus of the mid intestine
}

\author{
K D EGGLI, ${ }^{*}$ E LOYER, ${ }^{*}$ AND K ANDERSON $\dagger$
}

Departments of *Radiology and †Surgery, Children's Hospital National Medical Center, Washington DC, $U S A$

SUMMARY A case of malrotation of the mid intestine with volvulus is reported in an infant in whom the clinical and radiological appearances were unusual, and had led to a misdiagnosis of necrotising enterocolitis. If the correct diagnosis had been made earlier, operation might have saved the infant's life.

Volvulus of the mid intestine is a life threatening complication of intestinal malrotation with a high risk of mortality; it is common in infants who present with symptoms of malrotation. ${ }^{12}$

\section{Case report}

A $1200 \mathrm{~g}$ infant girl, small for gestational age, was born at 36 weeks' gestation. Apgar scores were 8 and 9 at one and five minutes. The infant did well for the first 3 days of life, feeding was begun at 24 hours of age, and was well tolerated. On the fourth day she developed bloody stools, abdominal distension, and increasing gastric residues. The initial radiographs of the abdomen showed elongated distended loops of bowel throughout the abdomen with pneumatosis cystoides intestinalis in the left upper quadrant, which were thought to indicate necrotising enterocolitis. Two later sets of radiographs also showed gaseous distension of the stomach and proximal duodenum that was unchanged over one day; the importance of the duodenal distension was not appreciated. The patient's clinical state worsened, and the intestine perforated, with evidence of pneumoperitoneum (fig). The dilatation of the duodenum was finally recognised and the diagnosis of probable volvulus of the mid intestine was made. At operation malrotation with volvulus of the mid intestine was found, with necrosis of most of the ileum and right and transverse colon. The infant died shortly afterwards.

\section{Discussion}

During normal embryological development the proximal duodenojejunal loop and the caecocolic loop independently complete counter clockwise $270^{\circ}$

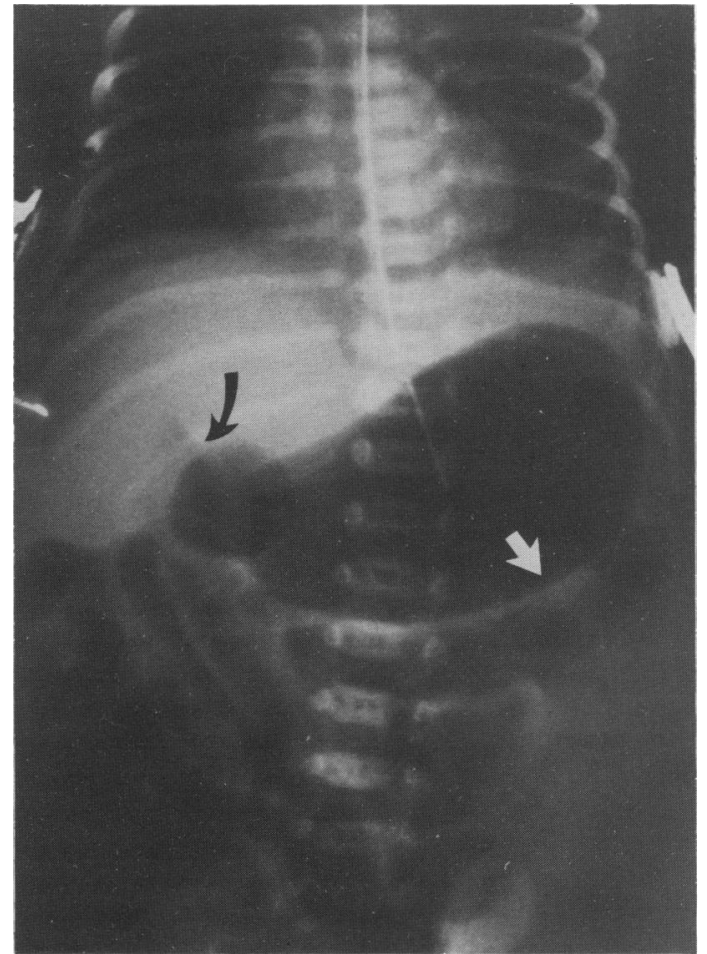

Figure Anterioposterior view of abdomen showing duodenum dilated out of proportion to remaining bowel. There is pneumatosis cystoides intestinalis in left upper quadrant (white arrow). Pneumoperitoneum has developed secondary to bowel perforation (black arrow on falciform ligament outlined by air).

rotations along the axis of the superior mesenteric artery. During this time the mesentery of the mid intestine acquires a wide base that extends from the ligament of Treitz to the caecocolic junction and is protected from volvulus and strangulation. Intestinal malrotation is a term referring to a wide range of embryological failures of rotation and fixation of the intestine that result in the mesentery having a narrower base than normal. Abnormal peritoneal 
bands representing indirect attempts at fixation cross the duodenum and are a source of obstruction.

Radiological diagnosis of malrotation depends on the identification of the abnormal position of the duodenojejunal junction. This junction should lie to the left of the spine and should reach a height that approximates to the junction of the first and second parts of duodenum. ${ }^{3}$

Though not all patients with malrotation have symptoms, those presenting with intestinal obstruction usually do so in the first three months of life. Younger infants who present in this way are at a higher risk of volvulus and bowel necrosis than patients who present when they are older. ${ }^{12}$

Once volvulus has occurred, the sudden onset of bilious vomiting is an almost constant finding, regardless of age. Before vascular compromise has occurred the clinical examination may be misleading, as the abdomen may be soft and non-tender between episodes of pain. As vascular compromise develops, abdominal distension, tenderness, bloody stools, and eventually hypovolaemia and shock develop. ${ }^{12}$

The pattern of abdominal bowel gas in volvulus of the mid intestine is variable and can be misleading. Mucosal ischaemia can lead to the development of pneumatosis cystoides intestinalis, as in the case presented here. This event, however, is rarely reported. In our review of published reports (including 382 cases of volvulus ${ }^{1245}$ ) only three such cases are mentioned. ${ }^{245}$ This gas pattern can be particularly confusing in a premature infant, in whom necrotising enterocolitis is the most common cause of pneumatosis cystoides intestinalis. The present case, and the three previously reported, illustrate this problem.

Proximal duodenal obstruction is a common finding with volvulus of the mid intestine, and the amount of gas distal to the obstruction (though usually reduced) may be variable. ${ }^{126}$ Dispropor- tionate duodenal dilatation, however, is not seen in straightforward necrotising enterocolitis. In our review of 96 films from 26 infants with uncomplicated necrotising enterocolitis, no duodenal dilatation was seen. Our patient's clinical signs of abdominal distention, non-bilious gastric residues and bloody stools-and the additional finding of pneumatosis cystoides intestinalis on abdominal radiograph-suggested a diagnosis of necrotising enterocolitis. It was the disproportionate amount of duodenal dilatation that finally suggested the diagnosis of malrotation.

\section{Conclusion}

Bowel necrosis caused by malrotation with volvulus of the mid intestine may mimic ischaemic bowel disease such as necrotising enterocolitis. Persistent distension of the proximal duodenum should suggest the correct diagnosis and lead to early contrast examination and surgical correction.

\section{References}

1 Potts SR, Thomas PS, Garstin WIH, McGoldrick J. The duodenal triangle: a plain film sign of midgut malrotation and volvulus in the neonate. Clin Radiol 1985;36:47-9.

${ }^{2}$ Berdon WE, Baker DH, Bull S, Santulli TV. Midgut malrotation and volvulus. Radiology 1970;96:375-83.

${ }^{3}$ Nussbaum A, Kirkpatrick JA. Malrotation of the midgut in infants and children. Part II. Contemporary Diagnostic Radiology 1983:6:4-5.

${ }^{4}$ Schorr S. Small-intestinal intramural air. Radiology 1963;81: 285-7.

5 Bunton GL, Durbin GM, McIntosh N, et al. Necrotizing enterocolitis. Arch Dis Child 1977;52:772-7.

${ }^{6}$ Kassner EG, Kottmeier PK. Absence and retention of small bowel gas in infants with midgut volvulus: mechanisms and significance. Pediatr Radiol 1975;4:28-30.

Correspondence to Dr KD Eggli, Department of Radiology, Children's Hospital National Medical Center, 111 Michigan Avenue NW, Washington DC 20010. USA.

Accepted 24 November 1988 